\title{
Diacronie
}

Studi di Storia Contemporanea

$N^{\circ} 27,3$ | 2016

Stato, costituzione e democrazia

\section{Philippe Poirrier (sous la direction de), La Grande Guerre. Une histoire culturelle}

\section{Federico Mazzini}

\section{Q OpenEdition}

Edizione digitale

URL: http://journals.openedition.org/diacronie/4217

DOI: 10.4000/diacronie.4217

ISSN: 2038-0925

Editore

Association culturelle Diacronie

Notizia bibliografica digitale

Federico Mazzini, «Philippe Poirrier (sous la direction de), La Grande Guerre. Une histoire culturelle », Diacronie [Online], №27, 3 | 2016, documento 22, Messo online il 29 septembre 2016, consultato il 24 septembre 2020. URL : http://journals.openedition.org/diacronie/4217 ; DOI : https://doi.org/10.4000/ diacronie. 4217 


\section{RECENSIONE:}

\section{Philippe POIRRIER (dir.), La Grande Guerre. Une histoire culturelle, Dijon, Éditions Universitaires de Dijon, 2015, 300 pp.}

a cura di Federico MAZZINI *

La storia culturale della Grande guerra in Francia è stata, negli ultimi due decenni, un argomento estremamente controverso. A partire dalla pubblicazione di 14-18 Retrouver la Guerre ${ }^{1}$ la storiografia accademica francese si è divisa, in un dibattito che è stato più volte raccontato ${ }^{2}$ ma raramente affrontato in Italia ${ }^{3}$, sulla questione del consenso delle truppe combattenti e del mondo civile alla guerra in corso e sul ruolo che la violenza ebbe nella costruzione di una cultura di guerra comune a tutti i soldati. Il dibattito si è presto cristallizzato nella contrapposizione tra gli studiosi dell'Historial di Péronne e quelli del CRID4, gli uni sostenitori di una storia culturale spesso diffidente verso le testimonianze dei soldati e impegnata a ricostruire il consenso "dal basso" alla violenza di guerra, gli altri intenti a provare, attraverso l'uso delle testimonianze e da una prospettiva di storia sociale, la preminenza della coercizione "dall'alto" e l'estraneità del corpo combattente agli scopi del conflitto e alle modalità con cui esso fu combattuto. La differenze interpretative e metodologiche tra Historial e CRID hanno portato a ulteriori dicotomie (storia culturale contro storia sociale,

\footnotetext{
1 BECKER, Annette, AUDOIN-ROUZEAU, Stéphane, 14-18, retrouver la Guerre, Paris, Gallimard, 2000.

2 PROCACCI, Giovanna, Alcune recenti pubblicazioni sulla «cultura di guerra» e sulla percezione della morte nel primo conflitto mondiale, in LABANCA, Nicola, ROCHAT, Giorgio (a cura di), Il soldato, la guerra, il rischio di morire, Milano, Unicopli, 2004, pp. 107-124; CESCHIN, Daniele, "Culture di guerra e violenza ai civili. Una "nouvelle histoire" della Grande Guerra?», in Ricerche di Storia Politica, XIII, 1/2010, pp. 43-56.

3 Tra le principali eccezioni: DEL NEGRO, Piero, FRANCIA, Enrico (a cura di), Guerra e culture di guerra nella storia d'Italia, Milano, Unicopli, 2011.

4 Il Collectif de Recherche International et de Débat sur la Guerre de 1914-1918 (CRID 14-18) nasce con sede a Craonne nel 2005, in diretta risposta al centro di Péronne e al «carattere spesso artificiale degli studi basati sui discorsi e sulle rappresentazioni». Cfr. Charte du CRID 14-18, URL: < http://crid1418.org/a_propos/charte_ini.html > [consultato il 19 luglio 2016].
} 
"centro" accademico contro "periferia", testimonianza contro cultura materiale, identità nazionale contro identità di classe) e a tensioni non solo storiografiche che sono evidenti, per quanto forse non immediatamente comprensibili, a qualsiasi studioso italiano che abbia partecipato a iniziative di uno dei due gruppi.

$\grave{\mathrm{E}}$ in questo contesto, da me qui fortemente semplificato, che si inserisce il volume curato da Philippe Poirrier, uno dei maggiori teorici francesi della storia culturale5. L'obiettivo del libro, esplicitato in introduzione, è quello di valorizzare, grazie all'attenzione suscitata dal centenario, i più recenti risultati della ricerca storicoculturale francese sulla Grande guerra e comunicarli ad un grande pubblico. Ma, come si affretta a specificare lo stesso Poirrier, è anche quello di offrire una storia culturale del conflitto inclusiva e interdisciplinare, non necessariamente in contrapposizione con la storia sociale, e capace di trarre dal dibattito sopra descritto ciò che di positivo esso ha saputo offrire alla storiografia: l'apertura di nuovi campi di ricerca, l'esplicitazione delle posizioni metodologiche dei singoli studiosi, l'individuazione dei punti ciechi e delle implicazioni etiche della scrittura della storia del conflitto.

La collocazione del volume all'interno del dibattito francese sulla cultura di guerra - o, per meglio dire, la sua esplicita volontà di non inserirsi in un'opposizione «intellettualmente sterile e oltraggiosamente schematica» ${ }^{6}$ - è ulteriormente specificata da Hervé Mazurel nel primo dei due saggi che compongono l'ouverture del libro. In esso Mazurel riassume la storia della storiografia culturale della guerra, importata in Francia per tramite degli scritti di Keegan e Mosse $^{7}$ e grazie ai toni taglienti e all'impostazione decisamente innovativa del già citato libro di AudoinRouzeau e Becker. L'autore racconta, in maniera ben più esaustiva di quanto sia possibile fare in questa sede, le ragioni tanto metodologiche quanto politicoistituzionali della contesa, ma sottolinea anche $\mathrm{i}$ punti in comune tra $\mathrm{i}$ due campi (entrambi guardano all'esperienza dei soldati nel tentativo di spiegare la permanenza al fronte di tutti gli eserciti a discapito delle condizioni della guerra di trincea) e, soprattutto, l'artificialità di alcune delle dicotomie che hanno caratterizzato il dibattito, in particolare la contrapposizione netta tra storia culturale e storia sociale come metodi di comprensione delle società passate.

5 POIRRIER, Philippe, Les enjeux de l'histoire culturelle, Paris, Seuil, 2004; ID., L'histoire culturelle: un "tournant mondial" dans l'historiographie?, Dijon, Éditions Universitaires de Dijon, 2008.

6 POIRRIER, Philippe (dir.), La Grande Guerre. Une histoire culturelle, Dijon, Éditions Universitaires de Dijon, 2015, p. 33.

7 In particolare, ovviamente, KEEGAN, John, Il volto della battaglia, Milano, Mondadori, 1978 [Ed. originale The face of battle, New York, Viking Press, 1976] e MOSSE, George L., Fallen Soldiers: reshaping the memory of the world wars, Oxford, Oxford University Press, 1990. 
Dopo l'ouverture il volume è diviso in due parti. La prima parte, Attori, prende in considerazione alcune figure professionali alle prese con il conflitto. Nella maggior parte dei saggi è evidente la volontà di restituire un quadro complesso e a volte contraddittorio, rifuggendo da nette dicotomie (consenso contro coercizione). La Grande Guerre des Journalistes di Laurent Martin dimostra per esempio l'ambiguità del ruolo delle pubblicazioni quotidiane nell'ambito del sistema di censura e di propaganda di guerra di contro all'immagine, spesso data per scontata, di un controllo onnipresente e uniforme. Nicolas Beaupré e Philippe Vatin si occupano rispettivamente della guerra di scrittori e artisti figurativi, dell'effetto che il conflitto ebbe sulle arti e sulla figura pubblica dell'artista e della rappresentazione artistica della guerra. I primi due saggi si concentrano sull'artista al fronte e nelle retrovie, evidenziando la sua integrazione all'interno delle strutture militari e di propaganda (i letterati come censori o propagandisti, gli artisti figurativi impegnati tanto nelle opere di mimetizzazione quanto nella "banalizzazione" della guerra illustrata), ma anche gli episodi di dissidenza e la progressiva ostilità alla guerra che caratterizza la rappresentazione letteraria e figurativa negli anni post-bellici ${ }^{8}$. Dal punto di vista tematico i saggi probabilmente più innovativi per un pubblico italiano sono quelli di Vincent Chambarlhac sulla guerra degli urbanisti, che lega il proliferare dei progetti e delle associazioni di urbanistica al «dovere patriottico di ricostruire» le città distrutte dal nemico, e quello di Clémentine Vidal-Naquet sulla guerra delle coppie. Quest'ultimo riassume ${ }^{9}$, in quello che ritengo l'intervento più complesso e stimolante della prima parte, le conseguenze «demografiche, economiche, sociali e intime» della separazione di 5 milioni di coppie (sposate) francesi tra il 1914 e il 1918 e, in particolare attraverso l'epistolografia, il processo di "accomodamento" dei rapporti coniugali alla guerra. La sezione è completata da approfondimenti sulla guerra degli sportivi (Paul Diestchy) e degli scienziati (Marie-Eve Chagnon). È significativo, data la struttura del libro e la composizione sociale dell'esercito francese ${ }^{10}$, che il ricco quadro della prima parte non includa una sezione su "La Grande Guerra dei contadini”: il punto cieco è probabilmente ereditato dalla storia culturale à la Péronne, che ha tradizionalmente dedicato scarsa o nulla attenzione alle culture contadine all'interno della "cultura di guerra”.

\footnotetext{
8 Di particolare interesse il riassunto dello studio statistico di Vatin che rileva, su un campione di 8500 opere, i temi e le allegorie ricorrenti della pittura di guerra francese e ne interpreta il significato culturale.

9 VIDAL-NAQUET, Clémentine, Couples dans la Grande Guerre. Le tragique et l'ordinaire du lien conjugal, Paris, Les Belles Lettres, 2014.

10 BARRAL, Pierre, Les campagnes, in AUDOIN-ROUZEAU, Stéphane, BECKER, Jean-Jacques (a cura di), Encyclopédie de la Grande Guerre 1914-1918, Paris, Bayard, 2014.
} 
La seconda parte del volume si occupa di Artefatti e oggetti culturali e si apre con due saggi sulla parola scritta, parlata e cantata: quello di Odile Roynette sull'evoluzione della lingua in seguito al conflitto (con una particolare attenzione verso la mobilitazione patriottica del linguaggio e i fenomeni di ibridazione determinati dall'esperienza di trincea) e quello di Anne Simon-Carrère sull'evoluzione delle canzoni di guerra (in particolare quelle diffuse dai café-concerts e dai teatri di guerra). Un interessante saggio di Benjamin Gilles è dedicato alla circolazione delle immagini di guerra e in particolar modo al rapporto tra censura, interesse commerciale dei periodici e fotografie scattate dai soldati. Tre interventi sono infine dedicati alla cultura materiale: Bertrand Tiller, storico dell'arte, lamenta il posto di secondaria importanza accordato all'arte e all'artigianato di trincea nella storia culturale della guerra (francese, ma lo stesso si può dire dell'Italia) e legge il processo di appropriazione che spinge i soldati a modellare creativamente i materiali della guerra tecnologica come un momento di resistenza capace di ridimensionare la teoria di un consenso popolare al conflitto. JeanCharles Capponier e Elsa Marguin Hamon raccontano la Grande Guerra del Patrimonio artistico francese, della sua salvaguardia e della sua strumentalizzazione politica; Daniel J. Sherman parla dei luoghi e dei monumenti della memoria.

La Grande Guerre. Une histoire culturelle non è soltanto una splendida introduzione "per il grande pubblico" alla storia culturale francese della guerra del 1914-18. L'attenzione che il libro dedica alle ultime evoluzioni metodologiche e tematiche del dibattito sulla cultura di guerra riveste un profondo interesse anche per gli storici, in particolare per chi ha osservato tale dibattito da questa parte delle Alpi. La storia culturale francese della prima guerra mondiale appare, nelle pagine del libro curato da Poirrier, non solo stimolante e innovativa, ma anche finalmente aperta a diversi approcci metodologici ed esiti interpretativi, capace di superare semplicistiche dicotomie e in grado di raccontare le ambiguità e le contraddizioni della "cultura di guerra”. 


\section{* L'autore}

Federico Mazzini, assegnista presso l'Università di Padova, si è occupato di scrittura contadina e cultura di guerra della Prima guerra mondiale, pubblicando nel 2013 Cose de laltro mondo. Una cultura di guerra attraverso la scrittura popolare trentina (Pisa, ETS, 2013). In questo momento si occupa della divulgazione scientifica attraverso riviste illustrate durante la Grande guerra e di storia culturale della tecnologia nel corso del Novecento.

URL: < http://www.studistorici.com/progett/autori/\#Mazzini >

\section{Per citare questo articolo:}

MAZZINI, Federico, «Recensione: Philippe POIRRIER (dir.), La Grande Guerre. Une histoire culturelle, Dijon, Éditions Universitaires de Dijon, 2015, 300 pp.», Diacronie. Studi di Storia Contemporanea : Stato, costituzione e democrazia, 29/09/2016,

URL:< http://www.studistorici.com/2016/09/29/mazzini_numero_27/ >

Diacronie Studi di Storia Contemporanea www.diacronie.it

Risorsa digitale indipendente a carattere storiografico. Uscita trimestrale.

redazione.diacronie@hotmail.it

Comitato di redazione: Jacopo Bassi - Luca Bufarale - Elisa Grandi - Antonio César Moreno Cantano - Deborah Paci - Fausto Pietrancosta - Alessandro Salvador - Matteo Tomasoni - Luca Zuccolo

Diritti: gli articoli di Diacronie. Studi di Storia Contemporanea sono pubblicati sotto licenza Creative Commons 3.0. Possono essere riprodotti e modificati a patto di indicare eventuali modifiche dei contenuti, di riconoscere la paternità dell'opera e di condividerla allo stesso modo. La citazione di estratti è comunque sempre autorizzata, nei limiti previsti dalla legge. 